\title{
Communication
}

\section{The Protein Kinase Activity of Phytochrome Functions in Regulating Plant Light Signaling}

\author{
Ah-Young Shin ${ }^{\dagger}$ Yun-Jeong Han ${ }^{\dagger}$, Pill-Soon Song and Jeong-Il Kim ${ }^{\dagger *}$ \\ ${ }^{\dagger}$ Department of Biotechnology and Kumho Life Science Laboratory, Chonnam National University, \\ Gwangju 500-757, Korea \\ ${ }^{\ddagger}$ Faculty of Biotechnology and Subtropical Horticulture Research Institute, Cheju National University, \\ Jeju 690-756 Korea
}

\begin{abstract}
Plant phytochromes, molecular light switches that regulate various aspects of plant growth and development, are known as autophosphorylating serine/threonine kinases. Although recent studies reveal that phytochrome autophosphorylation plays an important role in the regulation of phytochrome signaling through the control of phyA protein stability, the in vivo functional roles of phytochrome kinase activity in plant light signaling are largely unknown. Thus, it is necessary to investigate the detailed function of phytochrome as a protein kinase, which might include mapping of kinase domain on the phytochrome molecule, searching for substrates that could be phosphorylated by phyA, and in vivo functional analysis of the kinase activity with phytochrome mutants displaying reduced kinase activity. Our recent studies reveal that the kinase activity of phytochrome plays a positive role in plant light signaling. Therefore, we highlight the current knowledge about the functional roles of phytochrome kinase activity in the light signal transduction of plants, based on our recent results.
\end{abstract}

Phytochromes are red (R)/farred (FR) photoreceptors that regulate various aspects of plant growth and development. $^{1,2}$ In Arabidopsis thaliana, five isoforms of phytochromes have been known as phytochrome A (phyA) through phyE that may form homo- and

*To whom correspondence should be addressed.

E-mail: kimji@chonnam.ac.kr heterodimers. ${ }^{3}$ Among them, light-labile phyA is the primary photoreceptor responsible for mediating photomorphogenic responses in FR light, whereas light-stable phyB-phyE regulate $R$ light-mediated responses such as R-induced de-etiolation. Phytochromes are dimeric chromoproteins with covalently linked open tetrapyrrole chromophore phytochromobilin, and exist in two distinct but photo-interconvertible forms, red-light absorbing Pr and farred-light absorbing Pfr forms. They are biosynthesized as the $\mathrm{Pr}$ form in the dark, which can be phototransformed into the Pfr form upon exposure to red light. The Pr-to-Pfr phototransformation of phytochrome induces the highly regulated signaling network for photomorphogenesis in plants. ${ }^{4,5}$

Recent studies on the phytochrome-mediated light signaling discovered that phytochrome photoactivation impacts on the control of protein subcellular localization, ${ }^{6,7}$ transcription, ${ }^{8,9}$ protein stability, ${ }^{10,11}$ and protein phosphorylation. ${ }^{12}$ The photoconversion of cytoplasmic $\mathrm{Pr}$ to $\mathrm{Pfr}$ causes the translocation of phytochrome into the nucleus, which appears to be the early molecular event for phytochrome signaling. ${ }^{5,13}$ Photo-activated and nuclear-localized phytochromes trigger a transcription cascade that leads to the regulation of light-responsive genes, by interacting with transcriptional regulators, such as phytochrome interacting factors (PIFs) including PIF3. ${ }^{14}$ In addition, phytochromes have been known to interact with other downstream signaling components such as nucleotide diphosphate kinase 2 (NDPK2), ${ }^{15}$ and phytochrome kinase substrate $1(\mathrm{PKS} 1)^{16}$ and many more.

In addition to the transcriptional regulation by 
phytochrome, it has been known that protein phosphorylation and dephosphorylation play important roles in regulating phytochrome-mediated light signaling pathway. $^{12}$ For example, the phosphorylation of phytochromes accelerates its degradation, whereas its dephosphorylation slows the degradation. ${ }^{17}$ Furthermore, a number of transcription factors are known to undergo light-induced phosphorylation depending on their interaction with photoactivated phytochromes, which accelerates proteasome-mediated degradation of the factors. $^{18-20}$

Plant phytochromes are known as phosphoproteins long time ago. ${ }^{12,}{ }^{21}$ The site of phytochrome phosphorylation have been investigated with purified phyA from oat seedlings, and three serines, Ser8, Ser18, and Ser599, have been identified as the phosphorylation sites. $^{22}$ Among them, phosphorylation at Ser599 in the hinge region has been reported to prevent the interaction of phyA with its signal transducers such as NDPK2, ${ }^{23}$ suggesting that the hinge region of phytochromes serves as a phosphorylatable signal-modulating site which regulate protein-protein interactions between phytochrome and its signal transducers. Ser8 and Ser18 in the N-terminal extension region (NTE) have been identified as autophosphorylation sites of oat phyA, and the autophosphorylation provides a molecular mechanism for signal attenuation in phytochrome-mediated light signaling by accelerating the degradation of phyA. ${ }^{22,24}$ Since plant phytochromes are also known as serine/threonine kinases, ${ }^{21}$ phytochromes have been reported to directly phosphorylate several downstream signal transducers, such as cryptochrome 1 (Cry1) and auxin/indole-3-acetic acid (Aux/IAA) as well as PKS1. ${ }^{12}$ More recently, the Pfr form of phytochromes induces rapid in vivo phosphorylation of PIFs preceding degradation. $^{18,19}$ Furthermore, phyA mediates rapid Rinduced phosphorylation of Arabidopsis FHY1 and FHL in a low-fluence response, which might regulate the protein stability of FHY1 and FHL. ${ }^{20}$ Collectively, recent studies suggest that phosphorylation on phytochrome molecule is important for the regulation of phytochrome interaction with downstream signal transducers and also for the regulation of protein stability of phytochrome itself and its signal transducers, which might play an important role in fine -tuning phytochrome signaling.

Based on the observation that phytochromes are phosphoproteins, it has also been suggested the existence of protein kinase(s) that phosphorylate the phytochromes and protein phosphatase(s) that dephosphorylate them. However, despite extensive studies of phytochrome-interacting proteins, there is no report thus far of a protein kinase that can phosphorylate phytochromes. On the other hand, a few protein phosphatases have been reported as being able to interact with and specifically dephosphorylate phytochromes, including flower specific phytochrome-associated protein phosphatase (FYPP), ${ }^{25}$ phytochrome associated protein phosphatase 5 (PAPP5), ${ }^{17}$ and phytochromeassociated protein phosphatase type 2C (PAPP2C). ${ }^{26}$ All these protein phosphatases act as positive regulators in the phytochrome signaling, suggesting that the phytochrome signaling is regulated by phosphorylation and dephosphorylation in which phytochrome is autophosphorylated and then dephosphorylated by specific phosphatases. Based on these results, it has been suggested that phytochrome autophosphorylation decreases its signaling flux by reducing the amounts of phytochrome proteins, while phytochrome dephosphorylation increase the signaling flux by enhancing phytochrome interaction with its signal transducers and increasing its protein stability. ${ }^{18}$ However, the molecular mechanism about how the phosphorylation of these proteins by phytochromes affects the light signaling are still unknown, so further studies will be necessary to elucidate the signaling pathways related to the phytochrome kinase activity.

In order to clarify the phytochrome function as a protein kinase, we recently investigated the in vivo functional roles of kinase activity with oat phyA mutants displaying reduced kinase activity. According to the results of our study, transgenic plants expressing the phyA mutants with impaired kinase activity 
showed reduced photo-responsiveness to farred light. In addition, the degradation of PIF3 was significantly prevented in the transgenic plants under farred light compared with the transgenic plant of wild-type phyA. These results suggest that phytochrome kinase activity plays an important role in the regulation of phytochrome signaling through the control of PIF3 protein stability. Consequently, our results indicate that phytochrome kinase activity play a positive role in light signal transduction.

Overall, our current results provide a proposed model for the regulation of phyA-mediated light signaling (Fig. 1). In the dark, phyA proteins are synthesized and accumulated as $\operatorname{Pr}$ form in the cytosol, composed of autophosphorylated and dephosphorylated phytochromes due to the action of its own kinase activity of phyA and specific phosphatases. Upon illumination, the $\operatorname{Pr}$ form is photoactivated to the Pfr form that translocates from cytosol to the nucleus. At this point, the phosphorylated Pfr form of phyA is degraded via the ubiquitin/26S proteasome protein degradation pathway. This phyA degradation mechanism involving autophosphorylation could facilitate the efficient attenuation of the phyA signal. By contrast, phytochrome-associated protein phosphatases insure the stability of phyA protein for an enhanced light response in photomorphogenesis, resulting from a decline in the autophosphorylated phyA level. At the same time, the phyA proteins translocated into the nucleus act as a protein kinase, phosphorylating substrate proteins such as PIFs. The phosphorylated PIFs are then degraded, which de-repress the expression of light-responsive genes. In the dark, the expression of light-responsive genes is negatively controlled by PIFs at transcriptional regulation and by $\mathrm{COP} 1$ at proteolytic regulation, both of which suppress the start of photomorphogenesis in the dark. Upon light illumination (i.e., dark-to-light transition), COP1 is translocated into cytosol and PIFs are degraded by the kinase action of phyA, which release the suppression of the expression of lightresponsive genes, inducing photomorphogenic responses in plants. Therefore, the protein kinase activity of phyA plays a crucial role in controlling the number of active PIFs to elicit signaling events, and thus improve the response of plants to fluctuating light environments.

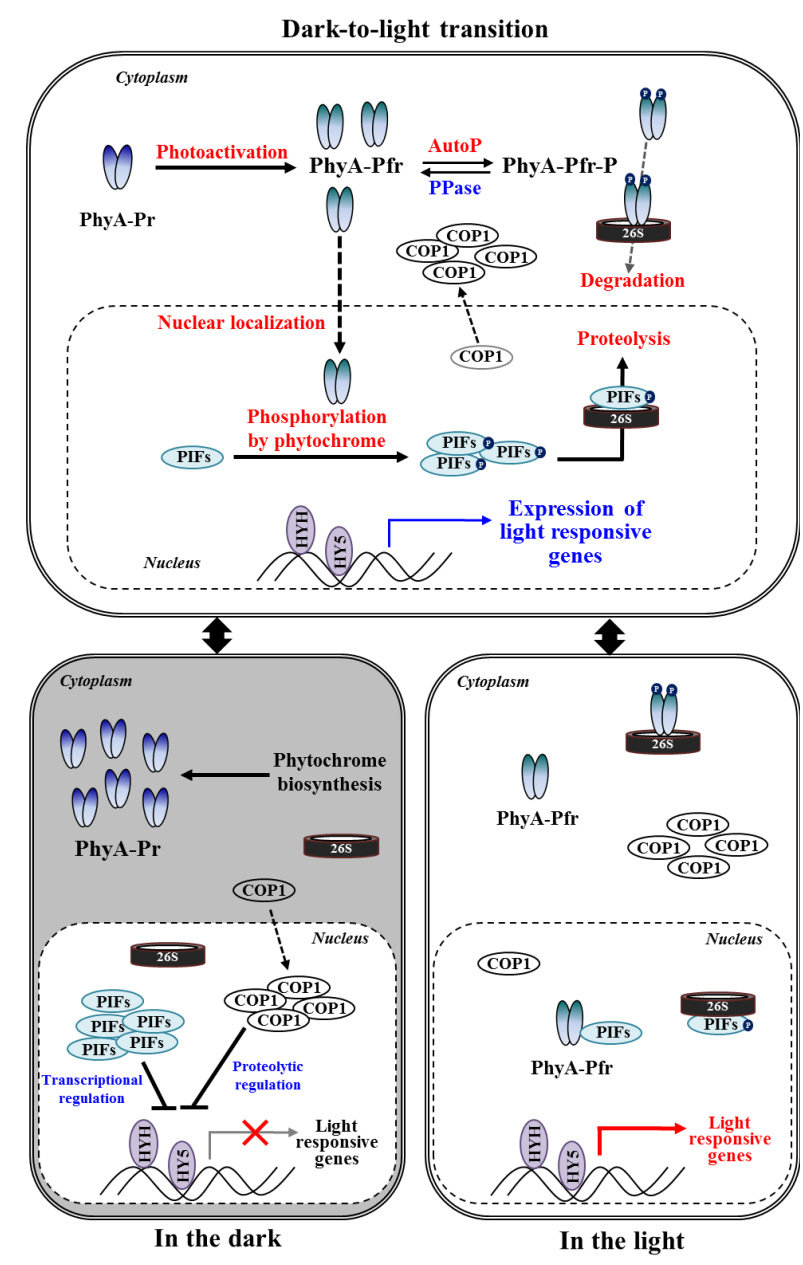

Figure 1. A proposed model for the molecular mechanism of phyA. In the dark, phyA are synthesized and accumulated as $\operatorname{Pr}$ forms in the cytosol. Light-responsive genes are not expressed because the photomorphogenesis-promoting transcription factors including two bZIP transcription factors, long hypocotyl 5 (HY5) $^{27}$ and HY5-homolog (HYH), ${ }^{28}$ are suppressed by PIFs. In addition, COP1 also negatively regulates the action of the transcription factors by promoting their degradation. In the dark-to-light transition, the $\mathrm{Pr}$ form is photoactivated to the $\mathrm{Pfr}$ form, which enter the nucleus. Then, they phosphorylate the substrate proteins such as PIF3, promoting its degradation by the ubiquitin/26S proteasome protein degradation pathway, which releases the suppression of the expression of light-responsive genes for photomorphogenesis and photosynthesis in the dark. Together, with the translocation of COP1 from the nucleus to the cytoplasm, the removal of negative regulators for light signaling (i.e., PIFs) by photoactivated phytochromes turns on the transcription of light-responsive genes, which allows plants to grow and develop optimally in the light environment. 
KEYWORDS : Phytochrome, Protein kinase,

Phosphorylation, Dephosphorylation, Light signaling

Received June 17, 2013; Accepted July 30, 2013

\section{ACKNOWLEDGEMENT}

This work was supported by Next-Generation BioGreen 21 Program (SSAC, grant no. PJ00949101), and by Basic Science Research Program through the National Research Foundation of Korea (NRF) funded by the Ministry of Education, Science and Technology (grant no. 2010-0012628).

\section{REFERENCES}

1. Rockwell, N. C.; Lagarias, J. C. Plant Cell 2006, 18, 4-14.

2. Bae, G.; Choi, G. Ann. Rev. Plant Biol. 2008, 59, 281-311.

3. Mathews, S.; Sharrock, R. A. Mol. Biol. Evol. 1996, 13, 1141-1150.

4. Quail, P. H. Nat. Rev. Mol. Cell Biol. 2002, 3, 85-93.

5. Chen, M.; Chory, J.; Fankhauser, C. Annu. Rev. Genet. 2004, 38, 87-117.

6. Nagatani, A. Curr. Opin. Plant Biol. 2004, 7, 708711.

7. Fankhauser, C.; Chen, M. Trends Plant Sci. 2008, 13, 596-601.

8. Jiao, Y.; Lau, O. S.; Deng, X. W. Nat. Rev. Genet. 2007, 8, 217-230.

9. Leivar, P.; Quail, P. H. Trends Plant Sci. 2011, 16, 19-28.

10. Hoecker, U. Curr. Opin. Plant Biol. 2005, 8, 469476.

11. Henriques, R.; Jang, I. C.; Chua, N. H. Curr. Opin. Plant Biol. 2009, 12, 49-56.

12. Kim, J. I.; Park, J. E.; Zarate, X.; Song, P. S. Photochem. Photobiol. Sci. 2005, 4, 681-687.

13. Lorrain, S.; Genoud, T.; Fankhauser, C. Curr. Opin. Plant Biol. 2006, 9, 509-514.

14. Ni, M.; Tepperman, J. M.; Quail, P. H. Cell
1998, 95, 657-667.

15. Choi, G.; Yi, H.; Lee, J.; Kwon, Y. K.; Soh, M. S.; Shin, B.; Luka, Z.; Hahn, T. R.; Song, P. S. Nature 1999, 401, 610-613.

16. Fankhauser, C.; Yeh, K. C.; Lagarias, J. C.; Zhang, H.; Elich, T. D.; Chory, J. Science 1999, 284, 1539-1541.

17. Ryu, J. S.; Kim, J. I.; Kunkel, T.; Kim, B. C.; Cho, D. S.; Hong, S. H.; Kim, S. H.; Fernandez, A. P.; Kim, Y.; Alonso, J. M.; Ecker, J. R.; Nagy, F.; Lim, P. O.; Song, P. S.; Schafer, E.; Nam, H. G. Cell 2005, 120, 395-406.

18. Al-Sady, B.; Ni, W.; Kircher, S.; Schafer, E.; Quail, P. H. Mol. Cell 2006, 23, 439-446.

19. Shen, Y.; Khanna, R.; Carle, C. M.; Quail, P. H. Plant Physiol. 2007, 145, 1043-1051.

20. Shen, Y.; Zhou, Z.; Feng, S.; Li, J.; Tan-Wilson, A.; Qu, L. J.; Wang, H.; Deng, X. W. Plant Cell 2009, 21, 494-506.

21. Yeh, K. C.; Lagarias, J. C. Pro. Natl. Acad. Sci. U. S. A. 1998, 95, 13976-13981.

22. Han, Y. J.; Kim, H. S.; Kim, Y. M.; Shin, A. Y.; Lee, S. S.; Bhoo, S. H.; Song, P. S.; Kim, J. I. Plant Cell Physiol. 2010, 51, 596-609.

23. Kim, J. I.; Shen, Y.; Han, Y. J.; Park, J. E.; Kirchenbauer, D.; Soh, M. S.; Nagy, F.; Schafer, E.; Song, P. S. Plant Cell 2004, 16, 2629-2640.

24. Han, Y. J.; Kim, H. S.; Song, P. S.; Kim, J. I. Plant Signal Behav. 2010, 5, 868-871.

25. Kim, D. H.; Kang, J. G.; Yang, S. S.; Chung, K. S.; Song, P. S.; Park, C. M. Plant Cell 2002, 14, 3043-3056.

26. Phee, B. K.; Kim, J. I.; Shin, D. H.; Yoo, J.; Park, K. J.; Han, Y. J.; Kwon, Y. K.; Cho, M. H.; Jeon, J. S.; Bhoo, S. H.; Hahn, T. R. Biochem. J. 2008, 415, 247-255.

27. Hardtke, C. S.; Gohda, K.; Osterlund, M. T.; Oyama, T.; Okada, K.; Deng, X. W. EMBO J. 2000, 19, 4997-5006.

28. Holm, M.; Ma, L. G.; Qu, L. J.; Deng, X. W. Genes Dev. 2002, 16, 1247-1259. 